\title{
Médiévales
}

Langues, Textes, Histoire

47 | automne 2004

Îles du Moyen Âge

\section{Îles, corsaires et pirates dans la Méditerranée médiévale}

Traduction Didier Boisseuil

Islands, Privateers and Pirates in the Medieval Mediterranean

\section{Pinuccia Franca Simbula}

\section{OpenEdition}

\section{Journals}

Édition électronique

URL : https://journals.openedition.org/medievales/500

DOI : $10.4000 /$ medievales.500

ISSN : 1777-5892

\section{Éditeur}

Presses universitaires de Vincennes

Édition imprimée

Date de publication : 1 décembre 2004

Pagination : $17-30$

ISBN : 2-84292-157-7

ISSN : 0751-2708

\section{Référence électronique}

Pinuccia Franca Simbula, « îles, corsaires et pirates dans la Méditerranée médiévale », Médiévales [En ligne], 47 | automne 2004, mis en ligne le 02 septembre 2006, consulté le 22 avril 2022. URL : http:// journals.openedition.org/medievales/500; DOI : https://doi.org/10.4000/medievales.500

Ce document a été généré automatiquement le 22 avril 2022.

Tous droits réservés 


\title{
îles, corsaires et pirates dans la Méditerranée médiévale
}

\author{
Traduction Didier Boisseuil \\ Islands, Privateers and Pirates in the Medieval Mediterranean
}

\author{
Pinuccia Franca Simbula
}

1 Qu'elles soient grandes et dispersées dans la mer Tyrrhénienne, petites, nombreuses et disséminées au Levant, les îles sont des nœuds essentiels des circuits commerciaux médiévaux, des bases économiques et militaires stratégiques, servant à contrôler et protéger les routes de navigation. Leur existence est liée, tout au long de l'histoire de la Méditerranée, à la course et à la piraterie. Forme légale de guerre pour l'une ou brigandage maritime pour l'autre, il était extrêmement difficile de les distinguer au quotidien, tout comme il était difficile d'empêcher les actes de pirateries menés par les navires qui avaient l'autorisation de pratiquer la course ${ }^{1}$. La course, progressivement disciplinée, se confond souvent avec la piraterie dont cependant - tout au moins de façon théorique - elle était nettement différenciée, ne serait-ce que parce qu'elle est susceptible d'entraîner des poursuites judiciaires. Au-delà de l'usage des termes, qui se clarifièrent progressivement, le recours à des combattants de métier sur les mers devient une pratique courante mais c'est une arme à double tranchant : contrôler des embarcations munies d'autorisations régulières et faire respecter les limites imposées par les concessions n'est pas toujours simple pour les autorités ${ }^{2}$. Navires de la flotte, corsaires privés et armateurs occasionnels sont souvent au centre des enquêtes et des accusations d'actes de piraterie.

2 Plus profondément, l'essor de la piraterie et de la course tient à la politique expansionniste des États en Méditerranée qui font des îles les confins de frontières mouvantes. L'activité des corsaires croît quand le pouvoir politique faiblit ou quand il le légitime en s'appuyant sur des pratiques et des normes guerrières qui, depuis le $\mathrm{XII}^{\mathrm{e}}$ siècle, font de la piraterie un moyen de combattre l'ennemi ${ }^{3}$. R. S. Lopez rappelle combien le conflit qui opposa Pise à Gênes «fut d'abord une lutte de corsaires », dont les protagonistes furent à la fois des aristocrates et des marchands, des membres de puissantes «consorterie » ou des armateurs autonomes, flanqués d'aventuriers et de 
mercenaires. La conquête de Bonifacio par les Génois, dans la dernière décennie du $\mathrm{XII}$ siècle, fut exclusivement «l'initiative, plus ou moins soutenue par l'État, de grands corsaires. $»^{4}$ Échappant aux Pisans, Syracuse tomba aux mains d'Alamanno da Costa, un noble génois, qui s'en empara avec l'appui de la République ligure, au nom de laquelle il en prit possession ${ }^{5}$.

Il s'agit, suivant les textes d'alors, de "pirates. » Jusqu'à la fin du XIII siècle, le terme est ambigu, car la distinction entre corsaires et pirates n'est pas encore établie. M. Balard souligne que dans l'Alexiade, Anne Comnène compare les expéditions des républiques maritimes italiennes en Syrie et Palestine à des opérations de piraterie contre l'Empire ${ }^{6} . \mathrm{Au} \mathrm{XII}{ }^{e}$ siècle, des commandants, qualifiés de pirates, se voient confier la défense des territoires byzantins ${ }^{7}$. C'est à une figure comme celle d'Enrico Pescatore qu'est inféodée, par le souverain sicilien, l'île de Malte, devenue entre la fin du XII et le début $\mathrm{du} \mathrm{XIII}^{\mathrm{e}}$ siècle, la base d'appui des offensives souabes et génoises. Pirate pour quelques-uns, courageux guerrier pour d'autres, Enrico navigue et combat sur les mers avec une flottille, au service de son roi et de sa patrie, Gênes; lesquels légitiment ses entreprises, parce qu'elles soutiennent leurs desseins politico-commerciaux. Entre 1206 et 1216 , il arrache la Crète aux Vénitiens qui le traitent de terrible pirate. Ses attaques visent à la fois les navires de Pise et de Venise, les cités rivales de Gênes. Son activité assure des bases solides à cette dernière dans la Méditerranée orientale, alors que la Sérénissime, confortée par la IV ${ }^{\mathrm{e}}$ croisade, se fait plus menaçante. Les vers du trouvère languedocien Peire Vidal s'opposent cependant aux affirmations vénitiennes; il décrit Enrico comme : "Larcs es et arditz e cortes, et estela dels Genoes, e fai per terra e per mar tots ses enemichs tremelar $»^{8}$. Ce n'est donc pas un brigand, mais un héros des mers qui inspire la terreur à ses ennemis, un combattant astucieux et valeureux qui est la gloire de Gênes. Ses relations avec la mère-patrie et le royaume de Sicile font de ses faits d'armes une guerre de course, et donc, légale.

4 Lointaines ou proches de la terre, les îles sont aussi des espaces idéaux pour tendre des embuscades aux navires de passage. En 1379, dans les eaux de Rhodes, coram Castro Rubeo, deux galères marseillaises, commandées par Nicolò Clavuoto et Antonio de Jérusalem, de retour de Beyrouth, sont surprises par quinze galères vénitiennes qui les dépouillent de leur précieux chargement de monnaies, de joyaux et de perles ${ }^{9}$. La même année, près de Majorque, une coque sur laquelle quelques marchands juifs avaient embarqué des draps, est attaquée par deux galères marseillaises, conduites par Pasquasio Arnion et Pietro Uguet et armées officiellement pour lutter contre les Sarrasins ${ }^{10}$. L'île d'Elbe est un repaire excellent pour surprendre les nombreux bateaux sortant de Porto Pisano; c'est là que le castillan Joan Rossell qui avait loué son embarcation à quelques marchands siennois, résidant à Pise et pour le compte desquels il avait chargé du grain en Sicile, est surpris par Giovanni Grimaldi, Génois à la solde du seigneur de Milan ${ }^{11}$.

5 Les îles offrent aussi des refuges : c'est dans les îles de la mer Tyrrhénienne qu'en 1404, le cavalier castillan Petro Niño pourchasse les corsaires de port en port. Parti de Toulon, à l'aube après une nuit de tempête, lui apparurent les îles tyrrhéniennes les plus septentrionales. Une fois reposés à Capraia, les équipages « prirent les rames et les galères et partirent à la recherche des corsaires, par toutes les îles petites. Ils allèrent à l'île de la Gorgone, à l'île de Pianosa et fouillèrent tous les ports dans les bouches de Bonifacio qui est en Corse. Là ils trouvèrent une embarcation aragonaise. Ils revinrent 
alors en Sardaigne, à Longosardo et à Alghero ", d'où ils continuèrent la chasse, explorant l'une après l'autre les côtes de l'île ${ }^{12}$.

6 Les fréquentes références, dans les sources documentaires, aux incidents qui se produisent autour des îles sont liées à l'intensité des trafics maritimes qui prennent appui sur elles, à la densité de la circulation des bateaux autour des ports et des passes, offrant aux corsaires et aux pirates les moyens d'agir plus facilement. Souvent cependant, les îles ne sont pas seulement les lieux où se déroulent les assauts, mais aussi ceux où s'arment les navires. La pauvreté de leurs ressources économiques maintes fois soulignée par l'historiographie - peut expliquer, mais en partie seulement que les îles soient devenues des repaires de corsaires et de pirates, au point d'être souvent décrite comme une disposition naturelle des habitants ${ }^{13}$. L'insertion des ports et des escales insulaires dans les circuits maritimes réduit sensiblement la distance entre les îles, les unit par des liens économiques, politiques, militaires et les place au cœur de réseaux d'échanges. La Crète, Chio, Negroponte, les Baléares, la Sardaigne, la Sicile comptent parmi ces îles qui constituent des nœuds commerciaux importants au Moyen Âge ${ }^{14}$.

7 En mer comme à terre, les États, d'un côté, imposaient des règles pour éliminer les désordres et les violences; de l'autre, la nécessité d'avoir à disposition des hommes prêts à combattre, attirés éventuellement par le butin, les obligeait à ne pas appliquer avec zèle les rigides dispositions normatives qu'ils prenaient ${ }^{15}$.

8 La navigation commerciale était la plus exposée aux coups de main et aux représailles inévitables lorsque les embarcations armées commettaient des razzie, qui, bien que réprouvées, n'étaient que faiblement condamnées ${ }^{16}$. L'absence d'escadre permanente favorisait l'emploi d'aventuriers de diverses origines; et le recours à la flotte marchande, afin d'affaiblir économiquement l'adversaire, était une des composantes de la guerre : les autorités publiques - dans les mondes ibériques, comme à Gênes, dans la mer Tyrrhénienne ou au Levant - percevaient d'ailleurs des droits sur les prises ${ }^{17}$.

9 Les petits spéculateurs, marchands ou armateurs, qui couraient les mers à la recherche de butin, étaient nombreux: les gouverneurs du royaume de Majorque concèdaient près de quatre-vingts licences d'armement à leurs sujets, entre 1375 et 1419. L'objectif des attaques est généralement spécifié, lorsqu'il s'agit de combattre les Maures ou des pays avec lesquels Majorque était en guerre. Suivant l'état des relations diplomatiques et pour ne pas compromettre de précaires équilibres, figurent alternativement les Grenadins, des sujets du roi de Fez, du sultan de Babylone, de Bône, de Bougie, de Constantine, de Pise ou de Gênes. Dans les autres royaumes, il en était de même. Des conditions étaient imposées: les sujets du royaume et les alliés-expressément mentionnés - doivent être respectés; les armateurs devaient verser une caution en raison des dommages que la Couronne pouvait se voir imputer si la capture était irrégulière ; des fidéjusseurs devaient souscrire ; le rayon d'action était parfois défini, pour maintenir les navires armés non loin des côtes qui devaient être protégées efficacement et contrôler les patrons ${ }^{18}$.

Dans ce panorama complexe, les îles n'eurent pas toutes la même importance comme base d'organisation de la course et de la piraterie. En raison des rivalités politiques, militaires et économiques, elles assurèrent des fonctions diverses.

11 La poussée irrépressible des républiques tyrrhéniennes et des Normands, à l'aube du deuxième millénaire, dilate la frontière maritime de la Chrétienté jusqu'à l'adosser sur les côtes nord-africaines et restreint les zones de confront avec l'Islam aux extrémités 
de la Méditerranée, vers les mondes ibérique et byzantin. Les îles sont en première ligne, point d'appui de l'avance occidentale et du contrôle des espaces maritimes. Sans solution de continuité, de l'aire tyrrhénienne jusqu'aux eaux du Levant, les différentes puissances, d'abord Pise et Gênes, puis les Vénitiens, les Byzantins, les Normands, les Angevins et les Catalans, élargirent leurs zones d'influence en imposant leur contrôle sur les îles, par le moyen de guerres incessantes et de renversements d'alliances. Dans ce cadre politique complexe, les îles délimitent les sphères d'affirmation sur la mer des différents États, signalent les étapes de l'expansion, offrent des plates-formes à la pénétration en Orient, constituent un glacis défensif aux influences économiques et politiques, un espace de rencontres culturelles et religieuses. Dans cette optique, ce que l'on retient davantage, c'est qu'elles assument une fonction de frontière, entre chrétiens et musulmans et entre puissances chrétiennes en guerre. Sur la mer, la guerre est faite parfois par de grandes expéditions mobilisant des flottes entières mais le plus souvent elle consiste en une multitude de rencontres, parmi lesquels les épisodes de la course sont déterminants ${ }^{19}$.

12 C'est un phénomène - tout comme la piraterie - qui concerne la vie des îles comme celle des ports continentaux, ainsi à Monaco et Piombino, sur la côte toscane, ou à Valence et à Alicante qui tirent d'importants profits de leurs positions de confins à l'instar des Baléares ${ }^{20}$. Même les ordres militaires, basés à Rhodes, dans les îles de l'Égée et à Malte, qui luttent, sans relâche, contre l'empire ottoman et le sultan d'Égypte, ne renoncent pas à des actes qui parfois s'éloignent même de la course ${ }^{21}$. En 1413, Rodrigo de Luna, neveu du pape Benoît XIII, chevalier de l'ordre de Saint-Jean de l'Hôpital de Malte et capitaine général de l'armée pontificale, arme, avec l'assentiment de son illustre parent, deux galères pour combattre les infidèles. Avec son équipage, composé de nombreux compatriotes, il parcourt les eaux nord-africaines et intercepte près de la Tunisie un navire génois, qui avait chargé ses marchandises à Grenade. Nonobstant la trêve en vigueur, il décide de s'emparer, de force, de la cargaison. À la suite d'un violent combat, durant lequel plusieurs marins ligures périrent, il capture l'embarcation et la traîne jusqu'en Sardaigne, dans le port complaisant de Cagliari ${ }^{22}$.

Parmi les îles de la Tyrrhénienne

13 L'imbroglio politique reflète le caractère confus des épisodes de course et explique les fonctions que les îles jouèrent en Occident, comme en Orient. La Corse en est un exemple : base convoitée sur les routes maritimes tyrrhéniennes, elle fut contestée par Pise et Gênes, avant l'arrivée des Catalano-aragonais. Les nombreuses ressources naturelles de l'île, la possibilité d'y fixer des établissements stables et surtout d'y contrôler des points côtiers de façon à garantir la sécurité de leurs propres navires et à créer un obstacle à ceux de leurs adversaires attirèrent les deux Républiques. Gênes parvint à s'en emparer, tout au moins formellement, car elle imposa péniblement son pouvoir à la noblesse locale. Le gouvernement génois se maintint au moyen d'une constante présence militaire, basée dans les deux colonies : à Bonifacio, conquise dès la fin du XII ${ }^{e}$ siècle, et Calvi, fondée en 1268, et sur des châteaux, stratégiquement placés et confiés à seigneurs locaux fidèles ou des châtelains d'origine ligure ${ }^{23}$. Pise écartée, l'aristocratie locale tenta vainement de trouver appui auprès des Aragonais, dans sa lutte éprouvante pour se débarrasser de Gênes ${ }^{24}$.

14 Bonifacio, par sa situation géographique et son autonomie politico-administrative est la clé de voûte de la domination génoise en Corse : colonie dotée de larges concessions pour favoriser son peuplement, c'est une escale et un port de transit, étroitement lié 
aux implantations ligures sur le continent et en Sardaigne occidentale. Qu'ils soient patrons, marchands ou marins, les Bonifaciens tentent fréquemment leur fortune comme corsaires. Les Génois avaient, en quelque sorte, reconnu et légalisé cette vocation des Corses. Dans les premiers statuts de la cité, il est fait obligation au podestat de réclamer une caution à tous ceux qui désireraient armer un navire pour la course. Suivant les conventions de 1321, les Bonifaciens décident d'ouvrir leur port à toute embarcation de corsaires moyennant le versement à la communauté d'une dixième partie du butin qui devait être débarqué et vendu sur place ${ }^{25}$. C'est là qu'à plusieurs reprises au milieu du $\mathrm{XV}^{\mathrm{e}}$ siècle, le tristement célèbre Battista Aicardo de Porto Maurizio, dit Scarincio, porta le fruit de ces razzie; en 1458, Gênes qui s'était maintes fois servie de lui prit ses distances et recommanda au podestat de ne plus accueillir celui qui était devenu un pirata publicus ${ }^{26}$.

Le champ d'action de ces armateurs locaux s'étend de la Sardaigne et la haute Tyrrhénienne jusqu'aux côtes de Provence. Toutefois, les prises réalisées, vendues au port, proviennent parfois d'eaux plus lointaines : à la fin du XIVe siècle, le marchand Guillem Sala est attaqué, à sa sortie du port, par une escadre de navires génois d'origine sicilienne et son embarcation, ainsi que sa cargaison de grains, sont vendues à Bonifacio $^{27}$.

16 Cette activité de course est importante en raison des investissements qu'elle représente et du nombre d'habitants impliqués. Grâce aux études de R. S. Lopez et de L. Balletto, les contrats de mutuo in panatica passés à Bonifacio ou à Gênes, à la fin du XIII siècle, sont bien connus; ils laissent percevoir le rôle important des corsaires dans la vie économique du «castello ${ }^{28}$. Un ensemble de ces actes, relatifs aux années 1244-1247, s'insère dans une phase extrêmement dure du conflit entre l'Église romaine et l'empereur Frédéric II, soutenu par Pise. Gênes, rangée au côté du pape, légitime les attaques contre les Pisans et les Siciliens, ainsi que contre les Musulmans de Barbarie, cible traditionnelle, en indiquant à ses sujets les objectifs à frapper. L'aperçu que ces documents nous offrent est extrêmement intéressant puisqu'il embrasse tout un ensemble de données : de l'aire d'action aux modalités de financement et de répartition des gains. Nombreux sont les Génois et les habitants de la Rivière ligure, qui, comme les Bonifaciens, entreprennent l'aventure. Une poignée d'actes révèle que plus d'une centaine de personnes, entre armateurs, propriétaires de navires, marins et changeurs a été intéressée par l'entreprise. Parmi les investisseurs de capitaux figurent de nombreux petits spéculateurs. Quelques-uns se démarquent, comme le notaire Ogerio Fornario qui, actif auprès de la cour de la cité et disposant plus aisément de quelques disponibilités financières, s'engage dans ces opérations fortement rentables, plus intéressantes que les activités commerciales classiques, ou comme son collègue Vivaldo Calignano, dont le nom revient fréquemment ${ }^{29}$.

17 Les autres Bonifaciens qui se prêtent à la course nous sont moins bien connus. La plupart sont des hommes de mer, quelques-uns disposent peut-être d'un peu de capital, venant sans doute d'activités commerciales. Il ne s'agit en aucun cas d'individus économiquement puissants. La course est faite par des hommes qui tentent de gagner leur vie ou de faire fortune, qui cherchent à joindre occasionnellement au travail de la terre les profits d'un butin rapide. C'est sans doute le cas de Nicoloso Peluco, serviteur au château de Bonifacio qui arrondit ses revenus en se consacrant au commerce et à la course ${ }^{30}$. 

corsaires et l'équipement des ports de l'île. Les villes sous domination ibérique, assiégées par mer et par terre, dépourvues de liquidités financières, manquant de victuailles et de renforts, obtiennent la possibilité d'armer et d'autoriser l'armement de navire de course, pour résister ou survivre. La capture ou le déroutement des embarcations de passage devint le mode de financement principal des établissements catalano-aragonais. Plusieurs aventuriers y contribuèrent avec passion : sans trop de formalités, corsaires et pirates trouvaient sur l'île un port sûr pour écouler leur butin provenant de toute la Méditerranée. De telles pratiques, qui durèrent plus d'un demisiècle, eurent des conséquences sur les activités commerciales et le rôle des places $\operatorname{sardes}^{36}$.

La politique de répression catalano-aragonaise fut extrêmement faible et variable, fluctuant en fonction des exigences militaires et des représailles sur les marchands catalans que les attaques ne manquaient pas de susciter. Entre 1350 et 1415, on ne dénombre pas moins de trois cents captures de navires. Ce seul chiffre montre l'ampleur du phénomène dont les principales victimes furent les Génois, les Provençaux, les Pisans, mais aussi quelques sujets de la Couronne qui avaient la charge-moyennant dédommagement - d'approvisionner les cités de Cagliari et d'Alghero, assiégées par le judicat sarde d'Arborea. Sous prétexte de défense, plusieurs ports armaient des navires et facilitaient le commerce des prises, avec la complicité et parfois la participation des officiers royaux et des seigneurs locaux. Cagliari et Alghero mettaient sur mer des embarcations et des équipages qui surveillaient les passages à proximité de la côte, poussant parfois à la recherche de proies jusqu'aux abords des rivages tunisiens, siciliens ou provençaux. Les seigneurs locaux et ceux des autres 
royaumes de la Couronne - comme le comte sicilien d'Augusta, Raymond de Moncade opéraient depuis les ports de Cagliari et d'Alghero, où ils amarraient leurs navires et enrôlaient les marins, approvisionnant les places-fortes du royaume et se garantissant de considérables profits ${ }^{37}$.

Gutierre Diez de Games a bien saisi la situation de l'île au tournant du XV siècle : «à cette époque, le roi d'Aragon n'avait plus en Sardaigne que Longosardo, Alghero, le château de Cagliari, parce tout le reste de l'île s'était rebellé. Le véritable maître était alors messer Branca, juge d'Arborea et seigneur de Monteleone. Les corsaires étaient accueillis et tolérés dans la mesure où ils apportaient soutien et aide quand cela était nécessaire; et c'est pour cette raison qu'ils étaient en sécurité et venaient en grand nombre $»^{38}$.

conquête définitive du royaume, entre 1410 et 1420, entraîna une baisse du nombre d'agressions et surtout la destruction des bases corsaires dans l'île. Ultérieurement, quelques seigneurs locaux, comme les Carroz ou les de Sena, ne renoncèrent pas totalement à des sorties avec leurs propres galères, surtout durant la campagne napolitaine d'Alphonse V. De nouveau, la guerre et le soutien accordé par la monarchie donnèrent aux attaques un semblant de légalitée ${ }^{39}$.

Les faits de course ou de piraterie et les évolutions politico-militaires étaient aussi étroitement corrélés en Sicile. Les Normands avaient assuré l'ordre sur les eaux en se servant, dans la seconde moitié du XII ${ }^{e}$ siècle, d'hommes de mer experts qu'ils rétribuaient : Margarito de Brindisi dont les sources documentaires rappellent les actes de piraterie en Orient; Guglielmo Grasso et Enrico Pescatore, autres figures de corsaires d'origine génoise qui servirent comme amiraux du royaume à la fin du XII et au début du XIII ${ }^{\mathrm{e}}$ siècle ${ }^{40}$.

Par la suite, à l'époque de Frédéric II, la course était relancée en Sicile et dans les îles mineures. Puis le contrôle étroit qu'exercaient les Angevins sur l'armement privèrent les corsaires de bases solides, tout au moins dans les principaux ports comme Palerme. La guerre des Vêpres et la lutte entre gibelins siciliens et guelfes napolitains entraînèrent l'apparition sur les mers de nouvelles attaques. Les navires provençaux, génois, pisans et vénitiens s'entre-attaquaient et à partir du XIV siècle, surgirent les Catalans qui dominèrent la scène ${ }^{41}$. À partir du milieu du siècle, en Sicile, l'effondrement du pouvoir royal permit, selon Henri Bresc, le développement des " seigneuries périphériques » qui trouvèrent dans la course un moyen d'accroître leurs ressources et de manifester leur autonomie. Cette activité atteignit son apogée au tournant du $\mathrm{XV}^{\mathrm{e}}$ siècle. Elle fut le fait des Chiaramonte, du comte Enrico Rosso, des Campolo, des Moncada, des Abbate, des Graffeo. De véritables seigneurs corsaires se fixèrent à la périphérie de l'île, à Mazzara, Pantelleria, Augusta, Lipari et Malte, traditionnels bastions du royaume qui fondait sa stratégie défensive sur la course. Les principales victimes restaient les Angevins, mais d'autres étaient assaillis dont les Génois. L'agressivité sicilienne était aussi dirigée contre les Hafsides qui relancèrent l'offensive et suscitèrent des razzie jusque sur la côte ${ }^{42}$.

27 À la fin du XIV siècle, l'affirmation du pouvoir catalano-aragonais et l'évolution des aristocraties locales mirent fin à la course proprement sicilienne, alors qu'augmentaient sensiblement le nombre de corsaires provenant du royaume ibérique, en particulier des Castillans au service de la Couronne et de la papauté avignonaise ${ }^{43}$. Ils portaient leur attention sur le commerce de grain destiné aux métropoles tyrrhéniennes; l'île et ses ports leur offraient assistance pour intercepter les 
embarcations chargées d'esclaves et d'épices. Cette activité s'intensifia dans la première moitié du XV $\mathrm{XV}^{\mathrm{e}}$ siècle, au moment où la lutte pour la domination de la Sicile était la plus violente, et lors de la conquête du royaume de Naples par Alphonse le Magnanime ${ }^{44}$.

Regard sur l'Orient

Plus complexe encore était la situation dans l'Orient byzantin. Une myriade d'îles entourait la terre ferme comme une membrane protectrice dont Rhodes et la Crète étaient les principaux éléments, Chypre la sentinelle avancée. Elles étaient les avantpostes de l'Empire, les bases sur lesquelles s'appuyait la flotte pour résister à la pression turque. La réduction des armements entraîna de façon irréversible l'effondrement du front après 1185 : corsaires et pirates s'insinuèrent dans les vides laissés par la flotte et jouèrent sur les désordres et la fragmentation de la périphérie maritime de l'Empire. Les îles subissaient attaques, occupations plus ou moins longues, saccages et rétorsions: elles étaient le théâtre d'affrontements sanglants entre chrétiens et musulmans, de rivalités complexes entre les flottes des puissances occidentales et ce même après la chute de Constantinople en $1453^{45}$.

Les conquêtes militaires des Latins et les concessions impériales qui leur étaient accordées dessinèrent de nouveaux confins, instaurèrent de nouvelles oppositions économiques et politiques. La présence de corsaires et de pirates dans les îles de la mer Égée était la manifestation la plus éclatante de la guerre, d'abord latente puis ouverte, entre Gênes et Venise pour l'extension de leur dominium, entre Souabes et Angevins pour la domination de la Sicile, entre Génois et Catalans. En toile de fond, se déroulait la guerre contre l'empire turc, avec lequel se conclurent des trêves périodiques. C'est dans ce bouillonnant Levant que «l'esprit de croisade, les conflits internationaux, les dissensions locales, les rivalités économiques offrirent au pillard le plus vil, suffisamment de prétextes pour relancer sa propre action, sans mettre en avant ses motivations personnelles $»^{46}$.

Venise chercha à limiter les dégâts causés par la piraterie en déployant un système de surveillance et de défense centré sur la Crète ${ }^{47}$. Le contrôle des armements privés, le règlement judiciaire des conflits occasionnés par la course, réduisit sensiblement le poids des Vénitiens dans les désordres du Levant: ils ne permettaient pas que se forment des "seigneuries corsaires ", même temporaires, dans les îles soumises à leur autorité. Les escadres vénitiennes n'hésitaient pas à frapper durement les corsaires et les pirates ou ceux réputés tels, réfugiés dans les îles, quitte à déployer un zèle parfois dénoncé par les Génois et les Catalans ${ }^{48}$.

31 C'était principalement à l'ombre de Gênes et de ses colonies orientales que se développaient la course et les faits de piraterie. Les établissements génois bénéficiaient d'une large autonomie par rapport à la mère patrie; les seigneurs d'Outremer alignaient leur politique sur des directives provenant de Gênes, mais leur statut ambigu - à la fois maître de seigneuries grecques et citoyen ligure - leur laissait une large autonomie. L'attitude de Gênes n'est pas moins ambiguë, qui se servaient d'eux sans réserve, niant les liens qui les unissaient et les responsabilités quand les complications internationales faisaient craindre des représailles. Des familles comme celle des Zaccaria, installée à Phocée et Chio, des Gattilusio, basée à Lesbos, Lemnos et Tasos, avec sa branche cadette fixée à Enos, Imbros et Samothrace construisirent sur cette double identité leur fortune et leur impunitée ${ }^{49}$. La défense des colonies orientales et la gestion des intérêts génois étaient assurées par une extraordinaire habileté 
entrepreneuriale, d'importantes capacités commerciales et des actions corsaires préjudiciables, par lesquelles les seigneurs accordaient un soutien naturel à leur patrie, à l'Empire byzantin et à leurs intérêts propres. Dans cette situation politique inextricable, il était facile de jouer sur l'équivoque, sur l'état de guerre latent, et la capture de chargement de grande valeur restait une perspective alléchante à laquelle il était difficile de renoncer. Une tentation à laquelle les Catalans n'ont pas résisté. Sans pouvoir profiter d'un système de colonies, ils utilisaient leur alliance avec Chypre et avec les Hospitaliers de Rhodes pour profiter de bases d'appui dans les îles, et profitaient de l'insertion progressive de la Sicile et de Malte à l'espace de leur domination ${ }^{50}$.

Les dommages causés à la navigation commerciale étaient graves, mais pas incommensurables. Les assurances maritimes étaient un instrument valide de protection ; la puissance des armements à bords et la navigation de conserve aidaient à se protéger des assauts, malgré l'augmentation inévitable des coûts de transport ${ }^{51}$. Cette réalité retentit dans les fonds documentaires, de façon dramatique. Elle tenait plus largement à la condition de frontière intimement liée à l'insularité. C'est à l'intérieur de ce monde que la guerre sur la mer, conçue principalement comme la guerre de course, avec sa prétendue efficacité, modulait les rythmes et l'intensité des rencontres méditerranéennes, offrant aux communautés, aux aventuriers, aux aristocrates et aux marchands un moyen de justifier leurs rapines. Les différents pouvoirs par leur faiblesse ou au contraire leur capacité d'encadrement ont conditionné le développement et les procédés de la course dans les îles. Directement ou indirectement liée aux relations internationales et à l'attitude des autorités, les armements corsaires et les attaques de piraterie finissaient par se mêler en un unique ensemble, surtout dans les îles qui se trouvaient aux confins de frontières incessamment remodelées où tout est incertain, où la volonté des pouvoirs lointains se superposait aux ambitions des particuliers. C'est à la fin du Moyen Âge, dans un climat de croisades, qu'elles finissent par acquérir le statut de bastion entre Occident chrétien et l'Islam.

\section{NOTES}

1. M. Fontenay-A. Tenenti, «Course et piraterie méditerranéennes de la fin du Moyen Âge au début du $\mathrm{xix}^{\mathrm{e}}$ siècle ", $X V^{e}$ Colloque International d'Histoire Maritime (San Francisco 1975), Paris, 1987, p. 78-136; M. Mollat, « De la piraterie sauvage à la course réglementée ( $\mathrm{xiv}^{\mathrm{e}}-\mathrm{xv}^{\mathrm{e}}$ siècle) », Mélanges de l'École française de Rome, 87, 1977, p. 591-609 ; Id., « Essai d'orientation pour l'étude de la guerre de course et de la piraterie (xiii ${ }^{\mathrm{e}}-\mathrm{xv}^{\mathrm{e}}$ siècles) », Anuario de Estudios Medievales, 10 (1980), p. 743-749 ; A. Tenenti, « Venezia e la pirateria nel Levante : 1300 c.-1460 c. », dans A. Pertusi dir., Venezia e il Levante fino al secolo XV, vol. I, Florence, 1973, p. 705-771.

2. Les plaintes des autorités sont fréquentes. Voir M. T. Ferrer, «Barcelona i la politica mediterrania catalana : el Parlament de 1400-1401 », dans M. G. Meloni et O. Schena dir., Atti del XIV Congresso di Storia della Corona d'Aragona (Sassari-Alghero 1990), Sassari, 
1995, vol. II, t. I, p. 427-443 ; M. D. López Pérez, « Piratería y corsarismo en el Mediterráneo occidental medieval ; el control de las actividades corsarias en Mallorca a finales del siglo xiv y principios del xv », dans Moll Blanes dir., VII Jornades d'Estudis Històrics Locals. La Mediterrània. Antropologia i Historia, I (Palma de Majorque 1988), Institut d'Estudis Baleàrics, Palma de Majorque, 1990, p. 173-203 ; A. Tenenti, « Venezia e la pirateria ", loc. cit., p. 708-746 ; G. Forcheri, Navi e navigazione a Genova nel Trecento. Il Liber Gazarie, Gênes, 1974, p. 35-43 ; J. Heers, Gênes au XV siècle, Paris, 1971, p. 223-227 ; M. Balard, "Course et Piraterie à Gênes à la fin du Moyen Âge », dans VIII Jornades d'estudis Histórics Locals : El comerç Alternatiu. Corsarisme i contraban (secoli XV-XVIII) (Palma de Majorque 1989), Palma de Majorque, 1991, p. 29-40 ; A. Roccatagliata, L'« Officium Robarie » del Comune di Genova (1394-1397), Gênes, 1989 ; E. Basso, Genova : un impero sul mare, Cagliari, 1994, p. 219-243.

3. M. Mollat, « De la piraterie sauvage », loc. cit., p. 591-609 ; Id., « Essai d'orientation pour l'étude de la guerre de course et de la piraterie (xiii ${ }^{\mathrm{e}}-\mathrm{xv}^{\mathrm{e}}$ siècles) », Anuario de Estudios Medievales, 10 (1980), p. 743-749 ; Id., La vie quotidienne des gens de mer en Atlantique, Paris, 1983, p. 156-177 ; P. F. Simbula, Corsari e pirati nei mari di Sardegna, Cagliari, 1993, p. 37-63 ; Ead., « I pericoli del mare : corsari e pirati nel Mediterraneo basso medievale », dans S. Gensini dir., Viaggiare nel Medioevo, Pise, 2000, p. 379-402.

4. R. S. Lopez, "Dieci documenti sulla guerra di corsa », dans R. S. Lopez, Su e giù per la storia di Genova, Gênes, 1975, p. 313-315.

5. Sur ce personnage, voir V. Vitale, Il comune del podestà a Genova, Milan-Naples, 1951, p. 150-155 ; G. Oreste, « Alamanno da Costa », Dizionario Biografico degli Italiani, vol. I, Rome, 1960, p. 574-575.

6. M. Balard, La Romanie génoise (XII -début du XV siècle), Rome, 1978, p. 587.

7. H. Ahrweiler, Byzance et la mer, Paris, 1966, p. 288-292. Un tableau complet des îles byzantines à la fin du xii ${ }^{\mathrm{e}}$ siècle est donné par É. Malamut, Les îles de l'Empire Byzantin, Paris, 1988.

8. Ces vers sont cités par D. ABULAFIA, « Henry count of Malta and his Mediterranean activities : 1203-1230 » dans Italy, Sicily and the Mediterranean, 1100-1400, Londres, 1987, p. 113. Sur Enrico et d'autres qui animent la mer entre la Sicile et le Levant, voir aussi M. Balard, op. cit., p. 470-481.

9. Archivo de la Corona de Aragón, Cancílleria, registro 1486, fo 95-96vº e 1488, fo 68-72 (désormais ACA, Canc. reg.).

10. ACA, Canc. reg. $1486, f^{\circ} 139 v^{\circ}-144$.

11. ACA, Canc. reg. 2011, fo $148 \mathrm{v}^{\circ}-149 \mathrm{v}^{\circ}$.

12. G. Diez de Games, El Victorial. Cronica de don Pero Niño, J. Sanz éd., Madrid, 1989, p. 110.

13. J. Heers, Gênes au XV siècle, op. cit., p. 227.

14. Voir F. Melis, I trasporti e le comunicazioni nel Medioevo, L. Frangioni dir., Florence, 1985, p. 179-223 ; M. Tangheroni, Commercio e navigazione nel medioevo, Rome-Bari, 1996, p. 443-85 ; U. Tucci, « Gli itinerari marittimi nel Medioevo » dans Viaggiare, op. cit., p. 39-58, et B. Dini, « I viaggi dei mercanti e il commercio internazionale nel Medioevo », ibid., p. 195-226.

15. Ph. Contamine, La guerre au Moyen Âge, Paris, 1980 (trad. it., La guerra nel Medioevo, Bologne, 1986, p. 328-337).

16. L'incidence de la course et de la piraterie sur les routes est bien documentée par la variation des primes d'assurances : M. Del Treppo, I mercanti catalani e l'espansione della Corona d'Aragona nel secolo XV, Naples, 1972, p. 416-423 et 483-522. 
17. Llíbre del Consolat de Mar, G. Colon-A. Garcia éd., Barcelone, 1987, vol. III, p. 50-51 ; Ordenanzas de las armadas navales de la Corona de Aragón, A. De Capmany éd., Madrid, 1987, p. 135-141 ; M. D. López Pérez, La Corona de Aragón y el Magreb en el siglo XIV (1331-1410), Barcelone, 1995, p. 626-637 ; P. F. Simbula, Pirati e corsari, op. cit., p. 39-55. 18. M. D. López Pérez, La Corona de Aragón, p. 557-812 et table 36, p. 595-633. 19. M. Tangheroni, « La prima espansione di Pisa nel Mediterraneo : secoli x-xii. Riflessioni su un modello possibile », dans G. Rosseti et G. Vitolo dir., Medioevo. Mezzogiorno. Mediterraneo. Studi in onore di M. Del Treppo, Naples, 2000, vol. I, p. 3-23 ; J. PRYOR, "At sea on the maritime frontiers of the Mediterranean in the High Middle Ages : the human perspective ", dans L. BALLETTO dir., Oriente e Occidente, Studi in onore di Geo Pistarino, vol. II, p. 1005-1034. G. PETRALIA, « Le "navi” e i “cavalli” : per una rilettura del Mediterraneo pienomedievale », Quaderni Storici, 103, fasc. 1, juin 2000, p. 201-222. 20. Bibliographie à jour dans J. Hinojosa Montalvo, « Pirateria y corso en la Edad Media valenciana », dans A. Sánchez Fernández dir., El Mediterraneo : un mar de pirats y corsarios, II Congreso Internacional de Estudios Históricos, Santa Pola, 2002, p. 91-114. 21. A. Tenenti, Venezia e la pirateria, loc. cit., p. 744-745 ; C. Jona, « Genova e Rodi agli albori del Rinascimento ", dans Atti della società ligure di Storia patria, LXIV, Genova, 1935, p. 67-154 ; A. Luttrell, The Hospitallers of Rhodes and their Mediterranean World, Aldershot, 1992 ; A. Rovere, « Un procedimento di rappresaglia contro Rodi (1388-1390) », dans Atti della Società Ligure di Storia Patria, Nuova Serie (1983), XXIII, fasc. II, p. 65-97.

22. ACA, Canc. Procesos, 103/6 (1414) ; S. Fossati Raiteri, « Il processo contro Rodrigo del Luna (1414) », dans Atti del $1^{\circ}$ Congresso storico Liguria-Catalogna, Genova 1969, Bordighera, 1974, p. 387-396.

23. L. Balletto, Genova, Mediterraneo Mar Nero, Gênes, 1976, p. 71-93 ; G. Petti Balbi, Genova e Corsica nel Trecento, Rome, 1976 ; J. A. Cancellieri, Bonifacio au Moyen Âge. Entre Gênes, Corse, Sardaigne et Méditerranée, Ajaccio, 1997.

24. J. A. Cancellieri, «Corses et Génoises : éléments pour une phénoménologie de la colonisation dans la Méditerranée médiévale ", dans M. Balard dir., État et colonisation au Moyen Âge et à la Renaissance, Lyon, 1989, p. 35-53 ; Id., " De la "Corse pisane" à la "Corse génoise" : remarques sur la portée structurelle insulaire de la bataille de la Meloria (1284) », dans Genova, Pisa e il Mediterraneo tra Due e Trecento (Atti della Società ligure di Storia Patria, Nuova Serie, vol. XXIV, fasc. II), Gênes, 1984, p. 569-583 ; voir également les articles réunis dans Medioevo. Saggi e Rassegne, 24 (2000) intégralement consacré aux relations avec la Couronne d'Aragon.

25. Id., « Dal trattato tra Genova e Sassari (1294) al trattato tra Bonifacio e Alghero (1386) », dans La Sardegna nel mondo Mediterraneo (Sassari 1978), Sassari 1981, vol. I, p. 261-275 ; G. PETTI BALBI, op. cit., p. 112-114 ; ID., « La pirateria nel Trecento : un episodio bonifaciano ", Medioevo. Saggi e Rassegne, 1983, 10, p. 29-31.

26. L. Balletto, «À travers la Méditerranée avec le pirate-corsaire Scarincio », dans Hatem Akkari Alif dir., La Méditerranée médiévale. Perceptions et représentations, ParisTunis, 2002, p. 153-169.

27. ACA, Canc., reg. 1487 , fo $129 \mathrm{r}^{\circ}$ e vo.

28. R. S. Lopez, Dieci documenti, op. cit., p. 318-319, 323-327 ; L. Balletto, Mercanti, corsari, op. cit., p. 35-95.

29. Sur les activités de ce notaire, voir L. Balletto, ibid., p. 69-71, 75-81, 89-93.

30. Id., ibid., p. 54-55, 91-95. 
31. E. Basso, " "Ferro, fame ac peste oppressa" : l'ammiraglio Bernat de Vilamarí e il blocco navale a Genova (1456-1458) », Anuario de Estudios Medievales, 24, 1994, p. 550-551; Id., « Pirati e pirateria nel Mediterraneo medievale : il caso di Giuliano Gattilusio ", dans A. Mazarakis éd., I Gattilusio di Lesbo (Mitilene 1994), Athènes, 1996, p. 349.

32. G. PetTI BALBI, op. cit., p. 112-114.

33. S. ORIGONE, "Sardegna e Corsica nel secolo XIV », dans Saggi e Documenti, I, Gênes, 1978, p. 369-388 ; ID., « Dal trattato tra Genova e Sassari (1294) al trattato tra Bonifacio e Alghero (1386) », dans La Sardegna nel mondo mediterraneo (Sassari 1978), Sassari, 1981, p. 261-275.

34. G. della Grossa, Chronique médiévale corse, M. Giacomo-Marcellesi et A. Casanova éd., Ajaccio, 1998, p. 244-245.

35. C. Carrère, Barcelona 1380-1462. Un centre económic en època de crisi, Barcelona, 1978, vol. I, p. 270-272 ; M. D. López Pérez, La Corona de Aragón, op. cit., p. 580-593 ;

P. F. Simbula, Pirati e corsari, op. cit., p. 37-45.

36. M. T. Ferrer i Mallol, « Barcelona i la politica », op. cit., p. 427-443 ; P. F. Simbula, op. cit., p. 94-110.

37. M. T. Ferrer i Mallol, « La conquista della Sardegna e la guerra di corsa nel Mediterraneo ", dans F. Carbonell, F. Manconi dir., I Catalani in Sardegna, Milano 1984, p. 35-40 ; P. F. Simbula, Corsari e pirati, op. cit.., p. 209-233.

38. G. Diez de Games, El Victorial, op. cit., p. 111.

39. M. Del Treppo, I mercanti catalani, op. cit., p. 499-506 ; A. Unali, Marinai, pirati e corsari catalani nel basso medioevo, Bologne, 1983, p. 121-136 ; Id., « Le flotte di Alfonso V d'Aragona destinate alle spedizioni nel Mediterraneo occidentale $(1420-23 ; 1432)$ : funzione, organizzazione, bottino ", Bullettino dell'Istituto Storico per il Medio Evo e Archivio Muratoriano, 97, 1991, p. 167-204.

40. Bibliographie et biographie sur ce personnage dans E. Basso, " Guglielmo Grasso », Dizionario biografico, LIX, Roma, 2002 ; G. Olgiati, « Gafforio », ibid., LI, Roma, 1998.

41. H. Bresc, "Course et piraterie en Sicile (1250-1450) », Anuario de Estudios Medievales, 10, Barcelone, 1980, p. 751-757 ; Id., Un monde méditerranéen. Économie et société en Sicile (1300-1450), Palerme, 1986, p. 338-345.

42. H. Bresc, ibid.; Id., " Course et piraterie », loc. cit.

43. Pour les royaumes catalano-aragonais, voir, outre la bibliographie déjà citée, la documentation en annexe de M. T. Ferrer Mallol, Corsarios castellanos y vascos en ele Mediterráno Medieval, Barcelone, 2000.

44. A. Unali, Marinai, pirati, op. cit., p. 121-136.

45. F. Thiriet, La Romanie vénitienne au Moyen Âge, Paris-Rome, 1959 ; G. Pistarino, Genovesi d'Oriente, Gênes, 1990, p. 283-370 ; B. Doumerc, « La colonisation des marchands ", dans M. Balard, A. Ducellier éd., Coloniser au Moyen Âge, Paris, 1995, p. 118-125 ; A. Ducellier, « Note sur les intérêts génois en mer Adriatique : le témoignage des archives ragusaines ", dans Oriente e Occidente, op. cit., cit., vol. I, p. 191-199 ; M. T. FERRER MALlOL, Corsarios castellanos, op. cit., p. 260-281.

46. A. TENENTI, op. cit., p. 738.

47. Jusqu'à mettre en place un contrôle étroit des armements privés : ibid., p. 750-751. Sur la Crète, $c f$. D. Jacoby, « Byzantine Crete in the Navigation and Trade Networks of Venice and Genoa », dans Oriente et Occidente, op. cit., I, p. 517-540 et C. A. Maltezou, «Creta fra la Serenissima e la Superba », ibid., I, p. 763-774.

48. A. Tenenti, op. cit., p. 724-737 ; M. Balard, La Romanie..., op. cit., p. 592-595. 
49. R. S. Lopez, Benedetto Zaccaria, ammiraglio e mercante nella Genova del Duecento, Florence, 1996 ; E. Basso, « Pirati e pirateria a Genova nel Quattrocento », dans La Storia dei Genovesi, Gênes, 1991, p. 327-351.

50. L. N. D'Olwer, L'expansió de Catalunya en la Mediterrània oriental, Barcelone, 1974 (3 éd.), p. 153-163 ; C. Carrère, barcelona, op. cit., p. 126-128 ; M. T. Ferrer Mallol, Corsarios castellanos, op. cit., p. 261-289.

51. M. Del Treppo, I mercanti catalani, op. cit., p. 483-522 ; A. Tenenti, op. cit., p. 751-761.

\section{RÉSUMÉS}

Les îles méditerranéennes ont souvent joué un rôle important dans l'organisation de la course et de la piraterie. Plus qu'une vocation, il s'agit d'un rôle imposé aux îles par leur condition de frontière maritime, par les conflits et la forme spécifique des guerres maritimes qui en font des lieux stratégiques de défense et de contrôle des routes commerciales et militaires. L'étude de quelques exemples permet de montrer comment la course et la piraterie se développent, dans les îles occidentales comme dans celles du Levant, dans un contexte de rivalités diffuses et de guerres ouvertes entre puissances chrétiennes et entre chrétiens et musulmans, qui les utilisent et les instrumentalisent. Consensus politiques ou incapacité des pouvoirs centraux à imposer des contrôles stricts des armements sont des facteurs déterminants dans la construction des espaces et des communautés insulaires qui se projettent avec agressivité sur la mer.

Islands, Privateers and Pirates in the Medieval Mediterranean. Mediterranean islands used to play an important part in the organisation of privateering and piracy. More than a vocation,this was a compulsory part imposed on the islands by their position of maritime borders, by conflicts and by the specific aspect of sea-wars that turned them into strategic places for defence and control over trade and military sea routes. The study of some examples show how privateering and piracy spread out in the western as in the Levant islands in a background of diffuse rivalry and open wars between Christian powers on the one hand and Christians and Muslims on the other hand, all of them using and manoeuvring them. Political consensus or the inability of central powers to establish strict control over weapons are determining factors in the construction of island spaces and communities that jutted out into the sea with aggressiveness.

\section{INDEX}

Keywords : islands, privateering, piracy, Mediterranean, Middle Ages

Mots-clés : course, piraterie, Méditerranée, Moyen Âge, îles

\section{AUTEUR}

PINUCCIA FRANCA SIMBULA

Dipartimento di Storia, Università di Sassari, v. le Umberto 5207100 Sassari, Italie 